\title{
Comprehensive Bridge Health Evaluation Method Based on Information Fusion
}

\author{
Li-ping $\mathrm{Li}^{1, a}$, Wen-xia Ding ${ }^{2}$ and Xiao-li Lu ${ }^{3}$ \\ ${ }^{1}$ Faculty of Engineering, China University of Geoscience, 430074 Wuhan, China \\ ${ }^{2}$ Hubei Communications Technical College, 430079 Wuhan, China \\ ${ }^{3}$ Wuhan University of Engineering Science, 430200 Wuhan, China
}

\begin{abstract}
In the bridge maintenance management decision, the health evaluation on bridge structure is far more than determination of abnormal index exceed fixed threshold, or evaluation on damage status and carrying capacity, the on-site human inspection should also be paid attention. In order to achieve more accurate and reliable health status evaluation result, it needs to take full advantage of various information and parameter. The D-S evidence information fusion was used in the paper to present comprehensive bridge health evaluation method. It fuses bridge operation status using abnormal status identification, bridge durability with residual life estimation, as well as human inspection rating, to arrive at health evaluation. The actual application examples to verify the method is also provided.
\end{abstract}

\section{Introduction}

The safety evaluation in bridge structural health monitoring is always one of the research focuses. Due to professional difference, the work of two main parts as information acquisition and safety assessment in structural health monitoring is completed by personnel of information science and mechanics science respectively. As a result, the existing structural safety assessment theory is limited to the mechanics field. Most methods depend on accurate structural model and known excitation system heavily. The safety assessment is achieved with reduction degree of structural stiffness [1,2]. The health safety assessment of bridge always regards structural damage identification as premise, namely firstly determine existence of damage and locate it, then evaluate safety status of structure with stiffness reduction.

In the existing bridge structure safety assessment methods, most depend on single parameter as the evaluating index, such as bridge damage or durability. As to complexity of bridge structure and environment, as well as randomness of measurement result, the evaluation evidence is always inadequate. It is impossible to accurately describe overall status of bridge structure, or even arrive at wrong evaluation result, so it has not high confidence for evaluating with single parameter [3, 4]. Some researchers proposed multi-parameter evaluation method and achieved satisfactory effect. Therefore, it is a valuable focus to perform overall evaluation on bridge status in accordance with acquired data from sensors as well as various parameter using information fusion method [5].

\footnotetext{
${ }^{\mathrm{a}}$ Corresponding author: 1lpok795@163.com.
} 
The paper combines various parameter of bridge health status with information fusion method to arrive at overall assessment on bridge. The rest of the paper is organized as follows: Section 2 introduces D-S evidence theory of information fusion; Section 3 gives comprehensive health evaluation method based on multiple information fusion; Section 4 and 5 present the computation example and the conclusion.

\section{D-S Evidence Theory}

Suppose $\Theta$ as identification framework. If the set function $m: 2^{\Theta} \rightarrow[0,1]$ meet $m(\phi)=0$ and $\sum_{A \subset \Theta} m(A)=1, m$ is called the basic confidence distribution on framework $\Theta$. As to $\forall A \subset \Theta, m(A)$ is the basic confidence distribution value.

In order to obtain total confidence of $A$, it needs to add the basic confidence distribution value of all subsets $B$ in $A$, and then represent it with confidence function. Assume $\Theta$ as an identification framework, the set function $B_{e l}: 2^{\Theta} \rightarrow[0,1]$ is confidence function if and only if it meets conditions as following:

(a) $B_{e l}(\phi)=0$;

(b) $B_{e l}(\Theta)=1$;

(c) To $\forall A_{1}, A_{2}, \cdots, A_{n} \subset \Theta$, there is

$$
B_{e l}=\left(Y_{i=1}^{n} A_{i}\right) \geq \sum_{i=1}^{n} B_{e l}\left(A_{i}\right)-\sum_{i<j} B_{e l}\left(A_{i} \cap A_{j}\right)+\cdots+(-1)^{n+1} B_{e l}(\overbrace{i=1}^{n} A_{i})
$$

Set $B_{e l 1}$ and $B_{e l 2}$ as two confidence functions on same identification framework $\Theta, m_{1}$ and $m_{2}$ as corresponding basic confidence distribution. Set $\sum_{A_{i} \cap B_{j}=\phi} m_{1}\left(A_{i}\right) m_{2}\left(B_{j}\right)<1$.

Then, the function defined by following is the basic confidence distribution of function $m: 2^{\Theta} \rightarrow[0,1]$,

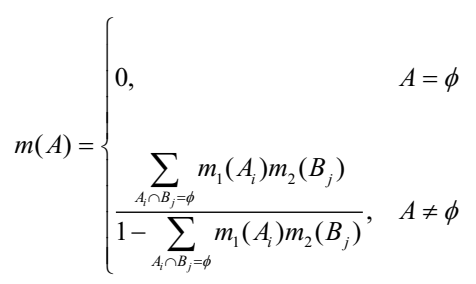

As to synthesis of multiple confidence functions, set $B_{e l 1}, \cdots, B_{e l \mathrm{n}}$ as confidence function on the unified framework $\Theta, m_{1}, \cdots, m_{n}$ as corresponding basic confidence distribution, if $B_{e l 1} \oplus \cdots \oplus B_{e l \mathrm{n}}$ exists and the basic confidence distribution is $\mathrm{m}$, as to $\forall A \subset \Theta, A \neq \phi$, there is:

$$
m(A)=k \cdot \sum_{\substack{A_{1}, \cdots, A_{n} \subset \Theta \\ A_{1} \cap \cdots \cap A_{n}=A}} m_{1}\left(A_{1}\right) \cdots m_{n}\left(A_{n}\right)
$$

where, $k=\left[\sum_{\substack{A_{1}, \cdots, A_{n} \subset \Theta \\ A_{1} \cap \cdots \cap A_{n} \neq A}} m_{1}\left(A_{1}\right) \cdots m_{n}\left(A_{n}\right)\right]^{-1}$.

It can be seen from (2) and (3) that the combination of multiple evidences has nothing to do with order. The computation of multiple evidence combination can be obtained by recursive computation of two evidences [6]. 


\section{Comprehensive Safety Assessment Based on Multiple Information Fusion}

\subsection{Evaluation Flow}

The evaluation flow of the proposed method is shown in Fig. 1.

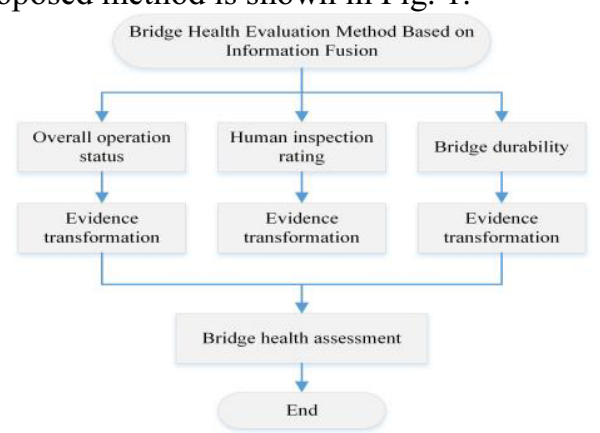

Figure 1. Multiple information fusion evaluation flow.

\subsection{Evidence Transformation of Evaluation Information}

As to comprehensive bridge health evaluation, its health status is a problem of data fusion, including statuses of health, unhealthy and uncertain. The paper constructs identification framework $\mu=\left\{A_{1}, A_{2}\right\}$. The $m\left(A_{1}\right)$ shows the structure is in safe state and $m\left(A_{2}\right)$ in unsafety status, so the uncertain status is $m(\mu)$. It is also an important in the information fusion to represent above evaluation results in the probability form and complete confidence distribution of different evaluation information.

\subsubsection{Evidence Transformation of Abnormal Status Evaluation}

The abnormal status evaluation on bridge usually determines whether some indicator exceed a fixed threshold. The abnormality degree of structure can also be obtained by computing indicator deviation from normal value. Due to complexity of structure itself and surrounding environment, it is quite difficult to determine a fixed evaluation threshold. If the inspection indicator is exactly equal to or close to the threshold, it is difficult to determine abnormal structure. The Exponentially Weighted Moving Average (EWMA) control chart was selected for bridge structure safety evaluation using monitoring information statistical analysis [7], Double control threshold alarming method was presented, namely preliminary alarm and safety alarm.

Referring to the EWMA control chart theory, the paper proposes double threshold abnormal status evaluation method, namely preliminary threshold $\delta_{\min }$ and alarm threshold $\delta_{\max }$.

To address the bridge structure abnormal determination, the system uses threshold as:

$$
\delta_{\lambda}=\bar{\lambda}+4 \sigma_{\lambda} .
$$

In order to improve accuracy of structural abnormal determination, as well as reduce the difficulty when the detection indicator is close to $\delta_{\lambda}$, the experiences can be used to set preliminary threshold $\delta_{\min }=\bar{\lambda}+4 \sigma_{\lambda}$, alarm threshold $\delta_{\max }=\bar{\lambda}+6 \sigma_{\lambda}$.

In accordance with the double threshold evaluation method and bridge management experiences, if the indicator obtained from abnormal diagnosis system is within the range of preliminary threshold, it may be considered $m\left(A_{1}\right)=0.8, m\left(A_{2}\right)=0.0$ and $m(\mu)=0.2$. When the detection indicator is beyond the alarm threshold, it can be considered $m\left(A_{1}\right)=0.0, m\left(A_{2}\right)=0.8$ and $m(\mu)=0.2$. If the 
indicator is between above thresholds, it can be considered $m\left(A_{1}\right)=0.33, m\left(A_{2}\right)=0.33$ and $m(\mu)=0.34$.

\subsubsection{Evidence transformation of bridge durability}

The bridge durability analysis can be obtained from durability of main structure. Boom is the key component to connect arch and road in the middle or low arch bridges. Its remaining life can be estimated from daily fatigue damage and cumulative damage degree. In order to evaluate fatigue durability of boom, the cumulative damage as well as relationship between remaining life and theoretical life should be used. The computation method considers not only cumulative damage of boom, but also effect of recent vehicle load on fatigue life. As the boom is force key of long span arch bridge, it directly affects the safety of arch bridge. The boom with the poorest durability can be used as basis to determine durability of bridge booms.

Represent the theoretical life is $N$ and remaining life obtained from fatigue estimation as $n$. The proportion relationship $\phi$ between estimated remaining life and theoretical remaining life as shown in (5) can be used to evaluate durability referring to classification boundaries in the bridge level maintenance specifications. The corresponding confidence distribution can also be determined.

$$
\phi=\frac{n}{N}
$$

In accordance with bridge level classification boundaries, the confidence distribution based on $\phi$ is shown in Table 1.

Table 1. Boom durability confidence distribution.

\begin{tabular}{|c|c|c|c|}
\hline$\phi$ & $m\left(A_{1}\right)$ & $m\left(A_{2}\right)$ & $m(\mu)$ \\
\hline$\phi \geq 0.88$ & 0.8 & 0 & 0.2 \\
\hline $0.88>\phi \geq 0.60$ & 0.7 & 0.2 & 0.1 \\
\hline $0.60>\phi \geq 0.40$ & 0.4 & 0.4 & 0.2 \\
\hline$\phi<0.4$ & 0 & 0.8 & 0.2 \\
\hline
\end{tabular}

\subsubsection{Evidence transformation of human inspection rating}

The traditional bridge health monitoring system is overly dependent on data acquisition system, but despise human inspection. As the health monitoring system only deploys sensors on some key components, so the sensor network cannot cover whole bridge. The uncovered sites are usually blind area for safe operation of bridge, while regularly necessary inspection may supplement above corners of health monitoring system. Therefore, it is very important of human inspection to health evaluation.

As to bridge health monitoring in the paper, human inspection is also designed, such as regular testing on linear between rib and deck, settlement of piers, as well as corrosion cracking of components. The confidence distribution of human inspection is always based on experiences. After each time of human inspection, the technical condition rating can be obtained from management department. The corresponding confidence distribution based on bridge rating is shown in Table 2 .

Table 2. Confidence distribution of human inspection status evaluation.

\begin{tabular}{|c|c|c|c|}
\hline Bridge Level & $m\left(A_{1}\right)$ & $m\left(A_{2}\right)$ & $m(\mu)$ \\
\hline First & 0.8 & 0 & 0.2 \\
\hline Second & 0.7 & 0.2 & 0.1 \\
\hline Third & 0.4 & 0.4 & 0.2 \\
\hline Fourth and fifth & 0 & 0.8 & 0.2 \\
\hline
\end{tabular}




\section{Comprehensive Evaluation Example}

Before safety assessment, it should firstly acquire needed data from corresponding database in accordance with evaluation needs. The evaluation amount can be obtained after information preprocessing. The preliminary result of bridge abnormal status, boom durability and human inspection will be transformed into corresponding evidence. The comprehensive assessment result can be achieved after D-S evidence fusion.

As the abnormal status diagnosis system is a near real-time system, the bridge is performed 6 time of abnormal diagnosis per minute. The boom lifetime computation updates once per day. Limited by factors, the update frequency of human inspection is uncertain. The maximum value of bridge status novelty indicator in each minute is used as basis for status scoring. The boom fatigue status rating and human inspection scoring are based on latest assessment.

Taking monitoring data of some bridge as example, the detection indicator data in early one hour is shown in Fig. 2.

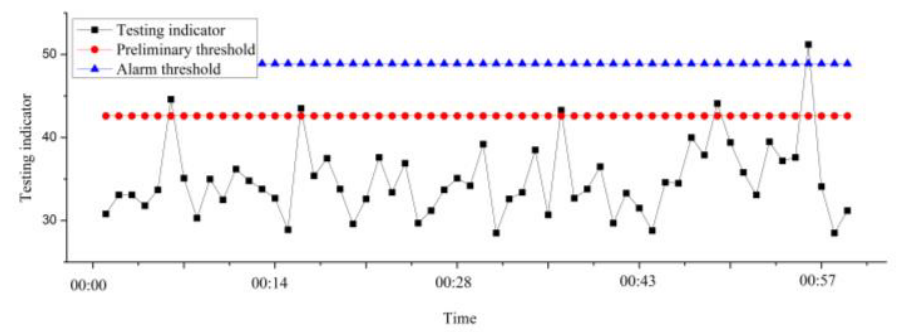

Figure 2. Overall status detection indicator.

As shown in Fig. 2, most detection indicators in the one hour are normal except for 5 points between preliminary threshold and alarm threshold, 1 point larger than the alarm threshold. It indicates that the bridge is normal in most of time. The sensor error or overload vehicles may temporarily impact on bridge structure. The monitoring indicator returns to normal after abnormal time points, which indicates the overall bridge structure is in good condition.

In accordance with computation result of daily damage, cumulative damage and remaining life of boom, the shortest remaining life of booms is 26.5 years. It can be regarded as the basis for boom durability determination. As the theoretical fatigue life of boom is 30 years and it has worked 1.25 years, the theoretical remaining life is 28.75 years. Based on (5),

$$
\phi=\frac{n}{N}=0.92>0.88 \text {. }
$$

It indicates the bridge boom durability is normal and the confidence distribution is $m\left(A_{1}\right)=0.8$, $m\left(A_{2}\right)=0.0$ and $m(\mu)=0.2$.

Based on latest bridge maintenance report and scoring result of management department, the bridge is in the second-class, so the confidence distribution is $m\left(A_{1}\right)=0.7, m\left(A_{2}\right)=0.2$ and $m(\mu)=0.1$.

Conduct D-S evidence fusion on abnormal status evaluation combining with boom durability and human inspection result, the overall safety assessment can be arrived as shown in Table 3, Table 4 and Table 5 .

Table 3. Overall bridge evaluation when the detection indicator is less than preliminary threshold.

\begin{tabular}{|c|c|c|c|}
\hline Evidence & $m\left(A_{1}\right)$ & $m\left(A_{2}\right)$ & $m(\mu)$ \\
\hline Abnormal status evidence & 0.8 & 0 & 0.2 \\
\hline Durability evidence & 0.8 & 0 & 0.2 \\
\hline Human inspection evidence & 0.7 & 0.2 & 0.1 \\
\hline
\end{tabular}




\begin{tabular}{|l|l|l|l|} 
D-S fusion evidence $m$ & 0.98 & 0.01 & 0.01 \\
\hline
\end{tabular}

Table 4. Overall bridge evaluation when the detection indicator is between preliminary threshold and alarm threshold.

\begin{tabular}{|c|c|c|c|}
\hline Evidence & $m\left(A_{1}\right)$ & $m\left(A_{2}\right)$ & $m(\mu)$ \\
\hline Abnormal status evidence & 0.33 & 0.33 & 0.34 \\
\hline Durability evidence & 0.8 & 0 & 0.2 \\
\hline Human inspection evidence & 0.7 & 0.2 & 0.1 \\
\hline D-S fusion evidence $m$ & 0.93 & 0.26 & 0.02 \\
\hline
\end{tabular}

Table 5. Overall bridge evaluation when the detection indicator is larger than alarm threshold.

\begin{tabular}{|c|c|c|c|}
\hline Evidence & $m\left(A_{1}\right)$ & $m\left(A_{2}\right)$ & $m(\mu)$ \\
\hline Abnormal status evidence & 0 & 0.8 & 0.2 \\
\hline Durability evidence & 0.8 & 0 & 0.2 \\
\hline Human inspection evidence & 0.7 & 0.2 & 0.1 \\
\hline D-S fusion evidence $m$ & 0.72 & 0.26 & 0.02 \\
\hline
\end{tabular}

According to actual siting inspection, the bridge is in good operation, which is consistent with evaluation result obtained from comprehensive bridge safety evaluation based on D-S evidence fusion method.

\section{Conclusion}

Using D-S evidence fusion method, the paper achieves comprehensive bridge safety assessment fusing operation status, durability and human inspection result. The accuracy of health safety assessment can be improved. However, the paper does not consider weight in the proposed method. In the later research, the D-S evidence theory should be thoroughly studied and weight of each evidence be studied, so as to further improve accuracy of safety assessment.

\section{References}

1. Abdelfateh Kerrouche, J. Leighton, W. J. O. Boyle, Y. M. Gebremichael, Strain Measurement on a Rail Bridge Loaded to Failure Using a Fiber Bragg Grating-Based Distributed Sensor System, IEEE Sensors Journal, vol. 8, no. 12, 2059-2065 (2008)

2. Victoria J. Hodge, Simon O'Keefe, Michael Weeks, Anthony Moulds, Wireless Sensor Networks for Condition Monitoring in the Railway Industry: A Survey, IEEE Transactions on Intelligent Transportation Systems, vol. 16, no. 3, 1088-1106 (2015)

3. Zhou Jianting, Huang Hanmin, Huang Shanglian, Chen Weiming, Remote Real-time Health Monitoring and Evaluation System for Long Bridge Structure, Proceedings of IMACS Multiconference on Computational Engineering in Systems Applications, vol. 2, 1751-1755 (2006)

4. Yu Chongchong, Zhang Jia, Tan Li, Wang Jinyan, Distortion data research of bridge structure health monitoring based on LS-SVM classification, Proceedings of 3rd International Conference on Information Sciences and Interaction Sciences (ICIS), 118-123 (2010)

5. F. Bruschetta, D. Zonta, C. Cappello, R. Zandonini, Fusion of monitoring data from cable-stayed bridge, Proceedings of IEEE Workshop on Environmental Energy and Structural Monitoring Systems (EESMS), 1-6 (2013)

6. Lan jin-hui, Ma Bao-hua, D-S evidence reasoning and its data fusion application in target recognition, Journal of Tsinghua University, vol. 41, no. 2, 53-55 (2001)

7. Qin Ping, Condition assessment based on cointegration and multivariate control chart for largespan bridges, Chongqing University, 45-46 (2015) 